\title{
EQUAÇÃO DE VON BERTALANFFY APLICADA AO CRESCIMENTO DE FRANGO COLONIAL*
}

\author{
MÁRCIA FERREIRA CARDOSO DE OLIVEIRA † \\ $\mathrm{E}$ \\ MARIA HERMÍNIA DE PAULA LEITE MELLO $\S$
}

\begin{abstract}
Resumo
A equação diferencial ordinária chamada de Equação de Von Bertalanffy é utilizada como modelo matemático para descrever o crescimento do frango colonial. A partir de dados estatísticos, deseja-se verificar se a Equação de Von Bertalanffy é um modelo matemático adequado para descrever o crescimento do frango colonial
\end{abstract}

\section{Introdução}

A produção de frangos de corte coloniais no Brasil está relacionada com a avicultura familiar. Nessas condições ela representa, muitas vezes, a viabilidade econômica das propriedades rurais, dos assentamentos da reforma agrária e de alguns pequenos municípios em vários estados brasileiros, onde pequenos produtores buscam um melhor desempenho na criação das aves visando a sua comercialização; contando, para esse fim, com recursos financeiros limitados e recursos naturais existentes na própria propriedade ou região em que vivem. (CRIAÇÃO de Frango Colonial, 2010).

Na criação alternativa de frango colonial, também comumente conhecido no Brasil como galinha caipira, de capoeira ou outras denominações regionais, as aves têm acesso à pastagem e sua alimentação inclui grãos, hortaliças, frutas, tubérculos e sementes. Como resultado deste sistema de criação, que difere da criação em confinamento de frango de corte, o frango colonial é de crescimento lento e tem uma carne mais escura e firme, com sabor acentuado e menor teor de gordura. (FIGUEIREDO et al., 2001).

\footnotetext{
*Palavras-chave: Modelagem Matemática, Equação de Von Bertalanffy, Crescimento de Frango Colonial, Método dos Mínimos Quadrados.

${ }^{\dagger}$ Especialização em Aprendizagem Matemática (cursando), UERJ; E-Mail: marciaferreira@ yahoo.com

§Prof. Associado IME/ UERJ; E-mail: mhermínia@ime.uerj.br
} 
O frango registrado como Embrapa 041 é um frango colonial resultado do cruzamento entre raças, já conhecidas em terreiros do Brasil, desde a década de 30. Para alcançar o peso do abate, em geral 2,5 quilos, o frango colonial necessita de mais tempo de crescimento, por volta de 12 a 13 semanas, enquanto que o frango de corte industrial é abatido com 7 semanas. (FIGUEIREDO, et al, 2001).

A motivação para estudar o crescimento do frango colonial através de um modelo matemático é devido à importância econômica da criação de frangos coloniais e da busca por um melhor desempenho na criação das aves visando à sua comercialização.

O modelo escolhido para descrever o crescimento do frango colonial foi a equação diferencial conhecida como equação de Von Bertalanffy, conforme sugestão de Bassanezi (1988, p. 82). Porém, para a determinação das constantes da equação de Von Bertalanffy, foram utilizados dados estatísticos do artigo de avicultura Criação de Frango Colonial (2010).

\section{A Equação de Von Bertalanffy}

O Modelo de Von Bertalanffy é um modelo matemático, desenvolvido pelo biólogo austríaco chamado Karl Ludwig Von Bertalanffy (1901-1972) e é utilizada para descrever o crescimento de vários tipos de animais.

O peso $\mathrm{p}(\mathrm{t})$ de cada espécie animal, em função do tempo t, pode ser expresso pela Equação de Von Bertalanffy:

$$
\frac{d p}{d t}=\alpha S-\beta p
$$

em que:

- S representa a superfície corporal do animal, que é dada em função do próprio peso;

- $\alpha$ é uma constante positiva, chamada de constante de anabolismo, que representa a taxa de síntese de massa por unidade de superfície do animal;

- $\beta$ é outra constante positiva chamada de constante de catabolismo, e representa diminuição ou perda de massa por unidade de massa. 
Assim, a equação de Von Bertalanffy estabelece que a taxa de variação instantânea do peso do animal, aumenta proporcionalmente à sua área corporal, porém tem um fator limitante, visto que, à medida que o peso do animal aumenta, este contribui para a diminuição da taxa de variação do próprio peso.

Precisamos estabelecer uma relação alométrica entre a área corporal do animal, no caso o frango colonial, e seu peso; isto é, apresentar a superfície corporal $S$ como uma função do peso do animal. Para isso, será utilizada uma forma geométrica para aproximar o corpo do frango, excluindo pescoço, cabeça e pés. Iremos supor que esse formato é um cubo. O peso do frango, $p=p(t)$, sempre positivo, é proporcional ao volume do seu corpo, $V=l^{3}, l$ sendo o comprimento linear. Assim, $p=k l^{3}$, para alguma constante de proporcionalidade $k, k>0$. Segue-se que $l=\frac{1}{k^{1 / 3}} p^{1 / 3}$. A área superficial do corpo (área corporal) será aproximada por $S=6 l^{2}$, o que implica $S=h p^{2 / 3}$, em que $h, h>0$, é a constante de proporcionalidade, que será incorporada à constante de anabolismo $\alpha$. O fator $2 / 3$ será o parâmetro alométrico.

Dependendo da espécie animal estudada, na relação alométrica $S=h p^{\gamma}$, onde $h$ é uma constante, o parâmetro alométrico, $\gamma$ pode assumir valores entre 0 e 1 para melhor se adequar à realidade (BASSANEZI, 2011, p. 141). Para a modelagem de crescimento de frango colonial será utilizada a relação alométrica $S=p^{2 / 3}$, assim como no estudo de crescimento de tilápias. (BASSANEZZI, 1988, p. 79- 83; ARAUJO; MÁRQUEZ, 2008).

Assim, o objetivo desse trabalho é o de verificar se o crescimento de frango colonial pode ser descrito através do modelo matemático:

$$
\frac{d p}{d t}=\alpha p^{2 / 3}-\beta p
$$

A equação (2) é uma equação do tipo Bernoulli com $n=2 / 3$. Para resolvê-la usamos uma mudança de variável $P=p^{1-(2 / 3)} \Rightarrow P=p^{1 / 3}$ a fim de obtemos uma equação linear de primeira ordem, com coeficientes constantes, não homogênea, na variável $P$, dada em (3).

$$
\frac{d P}{d t}+\frac{\beta}{3} P=\frac{\sigma}{3}
$$


A solução geral da equação (3) será:

$$
P(t)=\frac{\alpha}{\beta}+c e^{-(\beta / 3) t}
$$

Novamente, usando a mudança de variável $P=p^{1 / 3}$ obtemos a solução geral da equação de Von Bertalanffy (2) :

$$
p(t)=\left(\frac{\alpha}{\beta}+c e^{-(\beta / 3) t}\right)^{3}
$$

Como se trata de um problema aplicado, temos que levar em consideração o peso inicial $p_{0}=p(0)$ do frango. Desta forma, o modelo considerado para descrever o crescimento do frango colonial será:

$$
\left\{\begin{array}{c}
\frac{d p}{d t}=\alpha p^{2 / 3}-\beta p \\
p(0)=p_{0}
\end{array}\right.
$$

Usando a condição inicial dada, determinamos qual deverá ser o valor da constante $c$ da equação (5).

\section{Métodos estatísticos para a determinação das constantes de anabolismo e catabolismo}

A solução geral (4) foi encontrada através da aplicação de métodos teóricos de resolução de equações diferenciais. Porém, no problema real de crescimento de animais, como no caso do crescimento de frango colonial, o que dispomos são de dados estatísticos, obtidos de modo experimental. A partir deles devemos determinar as constantes $\alpha$ e $\beta$ (de anabolismo e catabolismo).

Iniciamos com a organização de uma tabela contendo dados experimentais dos pesos do frango colonial, medido em gramas, em função da idade, em semanas, obtidos em (CRIAÇÃO de Frango Colonial, 2010).

Consideramos na tabela outros dados que sejam pertinentes ao problema a ser estudado. Devido à natureza da equação diferencial do modelo matemático aqui tratado, vimos que é mais fácil trabalhar com a raiz cúbica do peso, $P(t)=(p(t))^{1 / 3}$. Assim, 
relacionamos também, na tabela, os valores de $P(t)$ e os seus valores na semana seguinte à semana t, ou seja $P(t+1)$.

Desejamos determinar uma função relacionando $P(t)$ e $P(t+1)$ e, para isso, faremos uso de métodos estatísticos. Não iremos tecer considerações detalhadas sobre os métodos estatísticos aqui empregados. Para uma referência especializada na área vide (ANDERSON et al., 2007).

Primeiramente, estabelecemos a correlação entre $P(t)$ e $P(t+1)$, utilizando o coeficiente de Pearson, que é um instrumento de medida de correlação linear. A correlação linear será tanto mais forte quanto mais próximo o coeficiente de correlação de Pearson (r) estiver de 1 ou de -1 , será tanto mais fraca quanto mais próximo estiver de zero. Se $r=1$ ou $r=-1$, então a correlação entre as variáveis é perfeita. Se $r=0$ então não existe nenhuma correlação. $\mathrm{O}$ sinal de $\mathrm{r}$ indica o sinal do coeficiente angular da reta ajustada (BASSANEZI, 2011, p.58-59).

O programa Microsoft Excel (2013), que já possui as funções e fórmulas estatísticas pré-programadas, foi utilizado para efetuar os cálculos da Tabela 3.1, com aproximações até a $6^{\text {a }}$ casa decimal; e, também para obter o gráfico de dispersão dado na Figura 3.1, cujos dados de entrada são os valores de $P(t+1)$ e $P(t)$ da Tabela 3.1.

Tabela 3.1 Dados do Frango Colonial

\begin{tabular}{|c|c|r|r|r|}
\hline dias & $\mathrm{t}$ - semana & $p(t)$ - peso em g & $P(t)=(p(t))^{1 / 3}$ & $P(t+1)$ \\
\hline 0 & 0 & 40,000000 & 3,419952 & 4,932424 \\
\hline 7 & 1 & 120,000000 & 4,932424 & 6,382504 \\
\hline 14 & 2 & 260,000000 & 6,382504 & 7,691372 \\
\hline 21 & 3 & 455,000000 & 7,691372 & 8,793659 \\
\hline 28 & 4 & 680,000000 & 8,793659 & 9,743476 \\
\hline 35 & 5 & 925,000000 & 9,743476 & 10,567218 \\
\hline 42 & 6 & $1.180,000000$ & 10,567218 & 11,292432 \\
\hline 49 & 7 & $1.440,000000$ & 11,292432 & 11,941848 \\
\hline 56 & 8 & $1.703,000000$ & 11,941848 & 12,531653 \\
\hline 63 & 9 & $1.968,000000$ & 12,531653 & 13,060859 \\
\hline 70 & 10 & $2.228,000000$ & 13,060859 & 13,541255 \\
\hline 77 & 11 & $2.483,000000$ & 13,541255 & 13,972736 \\
\hline 84 & 12 & $2.728,000000$ & 13,972736 & 14,362958 \\
\hline 91 & 13 & $2.963,000000$ & 14,362958 & 0,000000 \\
\hline
\end{tabular}

Fonte: Criação de Frango Colonial. Disponível em: < http://frangoc.blogspot.com.br/2010/04/criacao-de-frangocolonial.html> 
Figura 3.1 Diagrama de Dispersão entre $\mathrm{P}(\mathrm{t})$ e $\mathrm{P}(\mathrm{t}+1)$

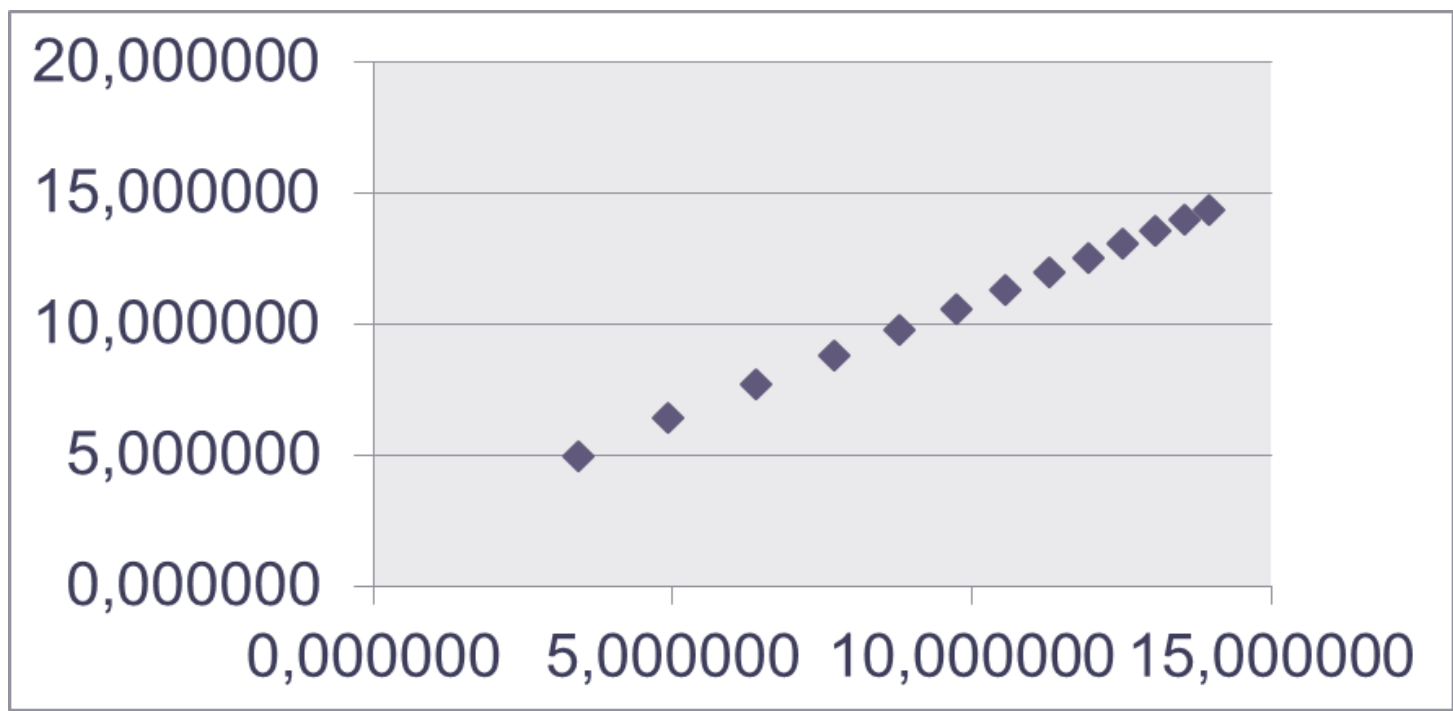

Para os dados da Tabela 3.1 acima, usando o programa Excel, a correlação encontrada entre $P(t)$ e $P(t+1)$ foi de 0,999929 que é muito próximo de 1 . Assim, observamos que a correlação entre as variáveis é muito forte, o que significa que a relação entre $P(t)$ e $P(t+1)$ é uma reta. Portanto, utilizaremos um ajuste linear, também chamado de método de regressão linear para estabelecer uma função entre $P(t)$ e $P(t+1)$. Isto é,

$$
P(t+1)=m P(t)+b
$$

Os coeficientes $m$ e $b$ da reta (7) podem ser encontrados usando o método dos mínimos quadrados. O programa Microsoft Excel (2013) permite encontrar esses coeficientes, diretamente, usando as funções ou fórmulas de Estatística préprogramadas. Desta forma obtemos a equação da reta:

$$
P(t+1)=0,885422 P(t)+1,968788 .
$$

A reta dada em (8) e será utilizada para a determinação de valores aproximados das constantes de anabolismo e de catabolismo, $\alpha$ e $\beta$ respectivamente, da equação de Von Bertalanffy (2).

A fim de determinar os valores aproximados de $\alpha$ e $\beta$ só precisamos da solução geral (4). De (4), temos que:

$$
P(t+1)=\frac{\alpha}{\beta}+c e^{-(\beta / 3)(t+1)} \Rightarrow P(t+1)=\frac{\alpha}{\beta}+c e^{-((\beta / 3) t+(\beta / 3))}
$$


Logo,

$$
P(t+1)=\frac{\alpha}{\beta}+c e^{-(\beta / 3) t} e^{-(\beta / 3)}
$$

Vamos considerar o sistema de equações:

$$
\left\{\begin{array}{l}
P(t)=\frac{\alpha}{\beta}+c e^{-(\beta / 3) t} \\
P(t+1)=\frac{\alpha}{\beta}+c e^{-(\beta / 3) t} e^{-(\beta / 3)}
\end{array}\right.
$$

Somando e subtraindo o termo $\frac{\alpha}{\beta} e^{-(\beta / 3)}$ ao segundo membro da equação (ii) e reagrupando os termos temos:

$$
\begin{gathered}
P(t+1)=\frac{\alpha}{\beta}+c e^{-(\beta / 3) t} e^{-(\beta / 3)}+\frac{\alpha}{\beta} e^{-(\beta / 3)}-\frac{\alpha}{\beta} e^{-(\beta / 3)} \\
P(t+1)=\left(c e^{-(\beta / 3) t} e^{-(\beta / 3)}+\frac{\alpha}{\beta} e^{-(\beta / 3)}\right)+\left(\frac{\alpha}{\beta}-\frac{\alpha}{\beta} e^{-(\beta / 3)}\right) \\
P(t+1)=e^{-(\beta / 3)}\left(c e^{-(\beta / 3) t}+\frac{\alpha}{\beta}\right)+\frac{\alpha}{\beta}\left(1-e^{-(\beta / 3)}\right)
\end{gathered}
$$

Pela equação $(i)$,

$$
P(t+1)=e^{-(\beta / 3)} P(t)+\frac{\alpha}{\beta}\left(1-e^{-(\beta / 3)}\right)=m P(t)+b
$$

Portanto, o coeficiente angular da reta, $m$, e o valor $b$, onde a reta intercepta o eixo y são identificados como:

$$
m=e^{-(\beta / 3)} \text { e } b=\frac{\alpha}{\beta}\left(1-e^{-(\beta / 3)}\right)
$$

Finalmente, achamos uma aproximação para o valor da constante $\alpha$ e uma aproximação para o valor da constante $\beta$, usando a equação da reta $P(t+1)=0,885422 P(t)+1,968788$, obtida em (8):

$$
\begin{array}{r}
0,885422=e^{-(\beta / 3)} \\
1,968788=\frac{\alpha}{\beta}\left(1-e^{-(\beta / 3)}\right)
\end{array}
$$

De (9), determinamos uma aproximação do valor da constante de catabolismo $\beta$. 
$0,885422=e^{-(\beta / 3)} \Leftrightarrow \ln (0,885422)=-\frac{\beta}{3} \Leftrightarrow \beta=-3 \ln (0,885422)$

Portanto $\beta \approx 0,365072$, onde o símbolo $\approx$ indica valor aproximado.

De (9), (10) e usando o valor aproximado de $\beta$, determinamos um valor aproximado da constante de anabolismo $\alpha$.

$$
\begin{aligned}
& 1,968788=\frac{\alpha}{\beta}\left(1-e^{-(\beta / 3)}\right) \Leftrightarrow 1,968788=\frac{\alpha}{\beta}(1-m) \Leftrightarrow \\
& 1,968788=\frac{\alpha}{\beta}(1-0,885422) \Leftrightarrow \frac{\alpha}{\beta}=\frac{1,968788}{1-0,885422}
\end{aligned}
$$

Como $\frac{\sigma}{\beta} \approx 17,182964$, temos , $\alpha \approx 6,273025$.

\section{Adequação do modelo matemático ao problema real}

É importante a verificar se o Modelo de Von Bertalanffy seria adequado para descrever o crescimento de frango colonial. Como já vimos neste trabalho, estamos interessados no problema de valor inicial (6):

$$
\left\{\begin{array}{c}
\frac{d p}{d t}=\alpha p^{2 / 3}-\beta p \\
p(0)=p_{0}
\end{array}\right.
$$

$\mathrm{O}$ valor inicial dado na Tabela 3.1 é $p_{0}=40 \mathrm{~g}$. Substituindo $t=0$ na solução geral (5), temos:

$$
p(0)=\left(\frac{\alpha}{\beta}+c e^{0}\right)^{3} \Rightarrow p(0)=\left(\frac{\sigma}{\beta}+c\right)^{3} \Rightarrow c=(p(0))^{1 / 3}-\frac{\sigma}{\beta} .
$$

Usando a mudança de variável, $P(0)=(p(0))^{1 / 3}$, encontramos a constante

$$
c=P(0)-\frac{\alpha}{\beta}
$$

Os valores aproximados de $P(0)$ e $\frac{\sigma}{\beta}$ são 3,419952 e 17,182964, respectivamente, portanto $c \approx-13,835818$ e o modelo matemático que adotaremos para descrever o peso do frango colonial será:

$$
p(t)=\left(17,182964-13,835818 e^{-0,121691 t}\right)^{3}
$$


Observamos que quando $t \rightarrow \infty, p(t) \rightarrow\left(\frac{\alpha}{\beta}\right)^{3}$; isto é, com o passar do tempo o peso do frango estabiliza, não ultrapassando $\left(\frac{\kappa}{\beta}\right)^{3} \approx 5.073,343002 \mathrm{~g}$.

Vemos na Figura 4.1 o gráfico da função $p(t)$ dada em (11), obtido através do programa Maple (Versão 9.5, 2004).

\section{Figura 4.1 Gráfico da Função $p(t)$}

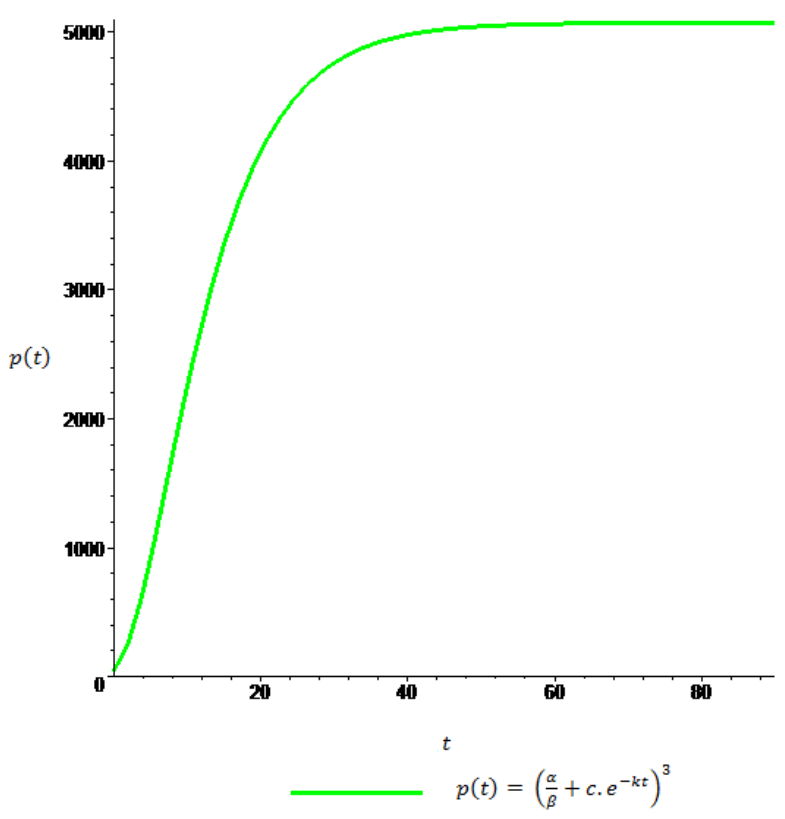

Para verificar se o modelo encontrado é adequado para descrever o problema real, vamos comparar os valores do peso do frango, $p(t)$, encontrados experimentalmente, que constam da terceira coluna da Tabela 3.1, com os valores encontrados utilizando a função dada em (11):

$$
p(t)=\left(17,182964-13,835818 e^{-0,121691 t}\right)^{3} .
$$

Observamos que poderá haver diferenças nos valores encontrados, visto que, para se obter a expressão da função (10), utilizamos valores aproximados das constantes pertinentes à formula. Assim, por exemplo, usando a fórmula da função (11), encontramos a condição inicial $p(0)=37,499349 \mathrm{~g}$ e não $40 \mathrm{~g}$. Porém para que o modelo seja adequado, precisamos encontrar valores para o peso do frango colonial que sejam bem próximos aos dados reais. Isto é o que, de fato, ocorre e pode ser observado na Tabela 4.1 abaixo. 
Apesar do modelo matemático dado em (11) ser uma aproximação do problema real, ele é bastante satisfatório para descrever o crescimento de frango colonial ou, pelo menos, o crescimento da amostra de frangos de que trata o artigo (CRIAÇÃO de frango colonial, 2010).

Tabela 4.1 Comparação Modelo Matemático e Dados Experimentais

\begin{tabular}{|c|c|c|}
\hline $\begin{array}{c}\text { t- } \\
\text { semanas }\end{array}$ & Valores de $p(t)$ em gramas \\
\hline 0 & 37,499349 & dados experimentais \\
1 & 120,000000 & 40,000000 \\
2 & 254,365893 & 120,000000 \\
3 & 435,326437 & 260,000000 \\
4 & 653,812768 & 455,000000 \\
5 & 899,645950 & 680,000000 \\
6 & $1.163,032237$ & 925,000000 \\
7 & $1.435,315037$ & $1.180,000000$ \\
8 & $1.709,276858$ & $1.440,000000$ \\
9 & $1.979,180091$ & $1.703,000000$ \\
10 & $2.240,666230$ & $2.228,000000$ \\
11 & $2.490,587608$ & $2.483,000000$ \\
12 & $2.726,816070$ & $2.728,000000$ \\
13 & $2.948,054010$ & \\
\hline
\end{tabular}

\section{Análise do modelo matemático}

Outra importância do modelo matemático seria a possibilidade de determinar, de uma maneira mais precisa, qual seria a melhor época para o abate da ave, bem como de ajudar no planejamento econômico para a criação do frango colonial, como verificar se vale a pena continuar a engorda da ave. 
Apesar da função $p(t)$ dada em (11) ser sempre crescente, tendendo a estabilizar com o passar do tempo, a análise da função derivada primeira, que mede a taxa de variação instantânea do peso, em função do tempo, é importante para verificarmos de que forma se dá esse crescimento do peso. Assim, passaremos ao estudo da função $p(t)$ dada em (11), analisando a sua derivada primeira e sua derivada segunda. Os cálculos e gráficos das funções derivadas foram obtidos por Oliveira (2015, p. 60-65), utilizando o programa computacional MAPLE (Versão 9.5, 2004).

Lembremos que a derivada primeira $\frac{d p}{d t}$ é a taxa de variação instantânea da função $p(t), t \geq 0$. Para a função $p(t)$ do modelo matemático encontrado, a derivada primeira será positiva pata todo $t \geq 0$. A sua fórmula não se anula para nenhum valor de $t \geq 0$. Na realidade, existe um ponto $t$, em que $t \approx-1,780397$, que anula a derivada primeira, mas por ser negativo, não interessa ao problema aplicado.

Analisando a expressão da derivada segunda, $\frac{d^{2} p}{d t^{2}}$, existe um único valor $T$, no domínio considerado, no qual ela se anula, $T \approx 7,247492$, sendo positiva para os valores de $t$ tal que $0 \leq t<T$ e negativa para $t>T$. Portanto, a derivada primeira, $\frac{d p}{d t}$, é estritamente crescente para $0 \leq t \leq T$ e estritamente decrescente para $t \geq T$ e o ponto $T$ é um ponto de máximo local, e também será um ponto de máximo absoluto, da função derivada primeira. Isto significa que, após $t=T$, se mantivermos o mesmo tipo e a mesma quantidade de alimentação, apesar do peso do frango aumentar, a variação de peso estará decrescendo, ou seja, continua-se tendo o mesmo gasto com a alimentação, mas o que o ganho de peso está se tornando cada vez menor. Manter o frango vivo, por muito tempo, com o mesmo tipo de alimentação pode não ser interessante economicamente, sendo esse um dos fatores que podem determinar a melhor idade para o abate da ave. Arredondando o valor de $T$, devemos abater o frango após à oitava semana. Mas para escolher exatamente qual seria a melhor semana para o abate da ave, outros fatores ainda devem ser analisados, como o peso médio que o frango deve ter para comercialização, se for o caso, sem que haja comprometimento do sabor e maciez da carne. Do ponto de vista matemático, apesar da taxa de variação instantânea do peso da ave estar decrescendo a partir de $t=T$, nota-se ainda um ganho significativo de seu peso; por isso, ainda vale a pena adiar um pouco o abate. Segundo Oliveira (2015), vemos que $\frac{d p}{d t}(T) \approx 274 \mathrm{~g} \mathrm{e} \frac{d p}{d t}(13) \approx 213 \mathrm{~g}$. Assim, na décima terceira semana a taxa de variação do peso é aproximadamente 213 g. Nos artigos especializados em 
avicultura, recomenda-se o abate com a idade mínima da ave de 85 dias, ocorrendo por volta da décima terceira semana (CRIAÇÃO de frango colonial, 2010, p. 3, 12-13). É interessante observar que a função derivada primeira tem um ponto de inflexão em $t=P$, em que $P \approx 13,783435$ e que $\frac{d p}{d t} \rightarrow 0$, a medida que o tempo passa. A partir do ponto de inflexão $P$ não compensa manter a ave viva.

Observamos que a função $p(t)$ também tem um ponto de inflexão, que é o ponto $t=T$.

A Figura 5.1 e a Figura 5.2 mostram os gráficos da derivada primeira e a derivada segunda, respectivamente, da função $p=p(t)$, obtidos através do programa MAPLE (Versão 9.5, 2004).

Figura 5.1 Gráfico da Primeira Derivada de $p(t)$

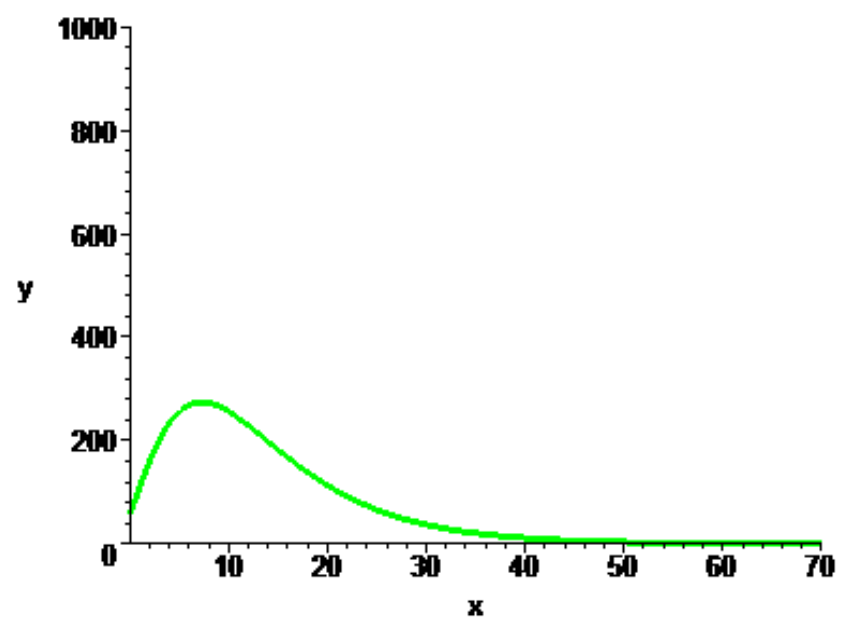

Figura 5.2 Gráfico da Derivada Segunda de $p(t)$

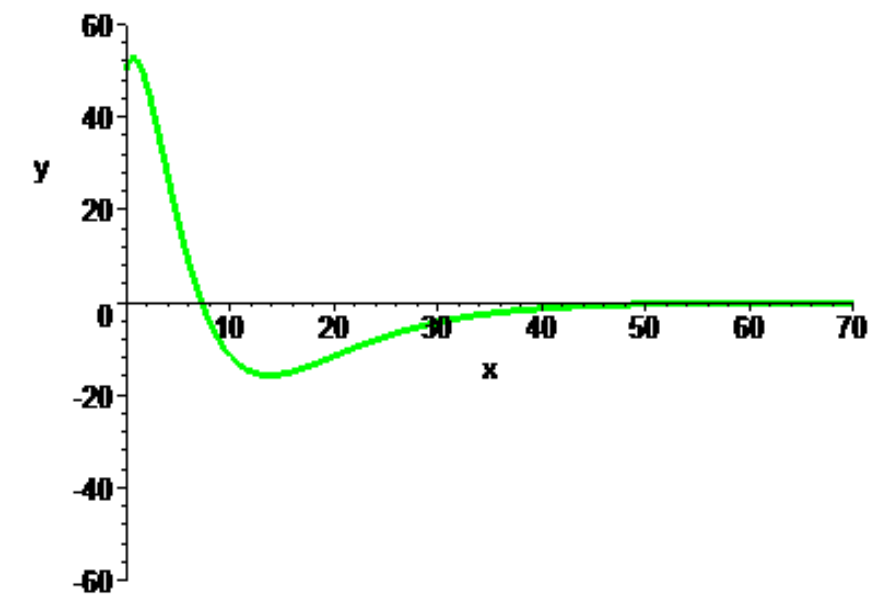




\section{Conclusão}

Com a modelagem matemática foi possível compreender melhor o processo de engorda do frango colonial e determinar, de uma maneira mais precisa, qual seria a melhor época para o abate da ave.

A equação de Von Bertalanffy mostrou ser um modelo matemático bastante adequado para descrever o crescimento do frango colonial.

\section{Referências}

ANDERSON, D. R.; SWEENEY, D. J. ; WILlIAMS, T. A.; Estatística Aplicada à Administração e Economia. Tradução: José Carlos Barbosa dos Santos. 2. ed., São Paulo: CENGAGE Lerning, 2007.

ARAUJO, J. C. de; MÁRQUEZ, R. M. G.: Modelos de Von Bertalanffy e Gompertz para Descrever os Parâmetros de Tamanho e Peso Médio de Tilápias. Cadernos do IME - Série Matemática. V. 20, p. 41-50, 2008.

BASSANEZI, R. C.; FERREIRA, W. C.: Equações diferenciais com Aplicações. São Paulo: Habra, 1988.

BASSANEZI, R. C.: Ensino-aprendizagem com modelagem matemática: uma nova estratégia. $3^{\mathrm{a}}$ ed.. $3^{\mathrm{a}}$ reimpressão, São Paulo: Contexto, 2011.

CRIAÇÃO de Frango Colonial. IN: Frango Caipira: Criação de Frango Colonial. 27 abr. 2010. Disponível em: < http://frangoc.blogspot.com.br/2010/04/criacao-de-frangocolonial.html > . Acesso em: 09 abr. 2013.

FIGUEIREDO, E. A. P.; ÁVILA, V. S.; ROSA, P. S.; JAENISCH, F. R. F.; PAIVA, D. P. de; Criação dos Frangos de Corte Coloniais Embrapa 041. IN: Instrução Técnica para o Avicultor. Embrapa, Suínos e Aves, 21. jun.2001. Disponível em:

<https://www.embrapa.br/suinos-e-aves/busca-de-publicacoes/-

/publicacao/443257/criacao-dos-frangos-de-corte-coloniais-embrapa-041. Acesso em: 2013.

OLIVEIRA, M. F. C. de: A importância da Modelagem Matemática na Educação. 2015. 65f. Trabalho e Conclusão de Curso (Licenciatura em Matemática). Instituto de Matemática e Estatística, Universidade do Estado do Rio de Janeiro, Rio de Janeiro, RJ, 2015. 